https://doi.org/10.15407/ujpe65.3.205

I.A. LYASHENKO, ${ }^{1,2}$ Z.M. LIASHENKO ${ }^{2}$

1 Technische Universität Berlin, Institut für Mechanik, FG Systemdynamik und Reibungsphysik, Sekr. C8-4, Raum M 122

(Strasse des 17. Juni 135, Berlin 10623, Germany; e-mail: i.liashenko@tu-berlin.de)

2 Sumy State University

(2, Rimskii-Korsakov Str., Sumy 40007, Ukraine)

\title{
INFLUENCE OF TANGENTIAL DISPLACEMENT ON THE ADHESION FORCE BETWEEN GRADIENT MATERIALS
}

\begin{abstract}
The influence of a tangential displacement on the strength of the adhesive contacts between gradient materials with different gradings of their properties has been studied. Variants with a controlled force (fixed load) and a controlled displacement (fixed grips) are considered. A relationship between the normal and tangential critical force components at which the contact is destroyed is obtained. It is valid within the whole interval of the gradient parameters, where the detachment criterium is obeyed. The optimal parameters at which the adhesive contact strength is maximum are determined. A case of detachment under the action of only the tangential force, i.e. when the normal force equals zero, is analyzed separately.

Keywords: adhesion, tribology, numerical simulation, method of dimensionality reduction.
\end{abstract}

\section{Introduction}

Functionally graded (gradient) materials are characterized by properties that gradually change in the material volume $[1,2]$. This effect can be achieved artificially by producing alloys in which the concentration of components is distributed non-uniformly. Gradient materials also exist in nature; these are bones, prickles of plants and animals, feathers of birds, plant stems, sea sponges, articular cartilages, and many other objects.

The overwhelming majority of natural graded materials have a biological origin. It is so because, first of all, the functioning of organs consisting of such materials is much better. This advantage was enhanced for various life forms in the course of their evolution. For example, many plants and animals use their prickles for the self-protection. Therefore, those prickles must be both stiff (to ensure the protection func-

(C) I.A. LYASHENKO, Z.M. LIASHENKO, 2020

ISSN 2071-0194. Ukr. J. Phys. 2020. Vol. 65, No. 3 tion) and elastic (otherwise, they can be broken by an external action). However, stiffness and elasticity are known to combine badly with each other. A solution to this problem consists in growing prickles with graded properties. Namely, the prickles have to be softer and more deformable at their sharp end, but they become stiffer and stiffer, as going along the prickle. If an alien object-guest moves along such a prickle, it will not feel pain at first, because the prickle is easily deformable and can withstand large deformations without being damaged at this stage. But if the motion along the prickle continues further, the latter becomes stiffer, and this circumstance does not allow the alien object to damage the organism which defends itself by means of such prickles without negative consequences for itself.

When applied in industry, gradient materials open a number of new possibilities, e.g., when developing devices ensuring a smooth dissipation of mechanical energy. In particular, they can be used as protective 
coatings at the sites, where somebody can fall from a high altitude. If a human body gets in contact with such a coating, the latter firstly offers minimal resistance because its elastic modulus is very low. But as the body "sinks" more and more into such a "floor", the counteracting force also increases. Knowing the kinetic energy of the body at the first contact, it is possible to calculate the thickness and the gradient index of the material that are required to avoid the appearance of supercritical stresses inside the body under which the internal organs or bones can be damaged.

Protective coatings of this kind can also be used in the production of safety helmets and the outer lining of bulletproof vests, in the manufacture of soles for sports sneakers, and so forth. One common thing is important for all those cases; this is the slowing down of the process of the mechanical energy transfer to the body that is protected by such materials.

Owing to a high demand for gradient materials in industry, there are many theoretical [3-5] and experimental $[6,7]$ works devoted to the research of such media, including the application of computer simulations as well [8-10].

The properties of gradient materials described above depend on the spatial coordinates, i.e. those materials are characterized by a spatial gradientness. Just materials of this type are studied in the presented work. However, if the specific features of gradient media are considered by an example of organs of living organisms, attention should be paid to that the gradient properties of those organs can also change in time. From this point of view, the gradient properties can be considered as functions of the time as well. So that, in some sense, we may talk not only about their spatial but also temporal gradients; in other words, about space-time characteristics.

The variation of the properties of gradient materials in time plays a very important role for living things. For example, a newborn hedgehog has prickles that are very soft along their entire length. Only with such prickles can it be formed in the womb of its mother without harming the maternal organism. After the birth, when the hedgehog continues to grow under natural conditions, the prickles become coarser and finally acquire protective functions. In order to describe such systems, where the gradient properties change in time, one should select, at every time moment, an appropriate coordinate function describing the gradientness and carry out a separate study, if necessary.

However, we may imagine another situation where the gradient properties of a material change very quickly in time. If those changes are rather rapid, they will affect the energy dissipation in the course of contact phenomena. The gradient properties of the material can be modified in time, e.g., with the help of an ac magnetic field, if the material contains magnetic particles. But the creation of such artificial materials possessing gradient properties with the space-time dependence is a long-term prospect. In this work, we study the case where the material properties (elasticity modulus) depend only on the spatial coordinates.

\section{Formulation of the Method of Dimensionality Reduction for Adhesive Contacts between Gradient Materials}

\subsection{Normal contact}

Let us describe the specific features of the method of dimensionality reduction (MDR), when it is used to analyze a contact between gradient materials indented only in the normal direction, i.e. without tangential displacements. We consider the case where the elasticity modulus of a material is given by the power function

$E(z)=E_{0}\left(\frac{z}{c_{0}}\right)^{k}, \quad-1<k<1$.

In order to describe a space-time-dependent gradientness, a time-dependent function has to be used instead of the constant $E_{0}$; e.g., it can describe the simplest case of Debye relaxation, $E_{0}\left(1-\mathrm{e}^{-t / \tau}\right)$. According to expression (1), this selection gives rise to the zero value of the elasticity modulus, $E(z)=0$, at the initial time moment (very soft prickles in the course of the intrauterine development of organism) and to a constant value [the value of the coefficient $E_{0}$ in formula (1)] at sufficiently long time intervals (adult organism).

It should be noted that a value of zero for the elasticity modulus has no physical meaning. However, it is possible to choose a time-dependent function for which the elasticity modulus has a non-zero value at the initial time moment. As concerning dependence (1), which gives the zero elasticity modulus at the material surface, the situation is somewhat more complicated because the formulas presented below are ob- 
tained just for formula (1), so that a change of this dependence will require the development of a new theory. In other words, we will consider a situation where the elasticity modulus at the material surface has a very small value. On the contrary, if the elasticity modulus at the material surface acquires values that are substantially different from zero, other techniques - like numerical simulation [11] - have to be used to study such systems.

In the case of elastic contact between two axially symmetric bodies, the MDR consists of two steps [1214]. Firstly, if the function $f(r)$ describing the original three-dimensional profile of the indenter is known (Fig. 1), it should be replaced by an equivalent one determined in the one-dimensional space, $g(x)$. For this purpose, the known transformation procedure is applied [15]:

$g(x)=|x|^{1-k} \int_{0}^{|x|} \frac{f^{\prime}(r)}{\sqrt{\left(x^{2}-r^{2}\right)^{1-k}}} \mathrm{~d} r$.

Below, we will describe, for simplicity, only a contact between an rigid parabolic indenter, $f(r)=$ $=r^{2} /(2 R)$, and the elastic half-space with gradient properties given by relation (1). Then the chosen function $f(r)$ gives rise to a one-dimensional representation in the form

$f(r)=\frac{r^{2}}{2 R} \Rightarrow g(x)=\frac{x^{2}}{(1+k) R}$.

At the next step [12-14], the elastic half-space should be replaced by an elastic foundation [16], as is shown in Fig. 1. This foundation consists of noninteracting springs with the normal, $k_{z}$, and tangential, $k_{x}$, stiffnesses dependent on the spatial coordinate $x$ in the one-dimensional space:

$$
\begin{aligned}
& k_{z}(x)=c_{N}(x) \Delta x=h_{N}(k, \nu) E^{*}\left(\frac{|x|}{c_{0}}\right)^{k} \Delta x, \\
& k_{x}(x)=c_{T}(x) \Delta x=h_{T}(k, \nu) G^{*}\left(\frac{|x|}{c_{0}}\right)^{k} \Delta x .
\end{aligned}
$$

Here, the constants (the effective elasticity, $E^{*}$, and shear, $G^{*}$, moduli at $k=0$ ) are determined by the relations

$E^{*}=\frac{E_{0}}{1-\nu^{2}}=\frac{2 G_{0}}{1-\nu}, \quad G^{*}=\frac{4 G_{0}}{2-\nu}$,

ISSN 2071-0194. Ukr. J. Phys. 2020. Vol. 65, No. 3 so that

$G^{*}=E^{*} \frac{2-2 \nu}{2-\nu}$.

The functions $h_{N}(k, \nu)$ and $h_{T}(k, \nu)$ in Eqs. (4) and (5), respectively, are determined by the formulas $[13,14]^{1}$

$h_{N}(k, \nu)=\frac{2(1+k) \cos \left(\frac{k \pi}{2}\right) \Gamma\left(1+\frac{k}{2}\right)}{\beta(k, \nu) \gamma(k, \nu)}$,

$h_{T}(k, \nu)=\frac{2 \beta(k, \nu)(2-\nu) \cos \left(\frac{k \pi}{2}\right) \Gamma\left(1+\frac{k}{2}\right)}{(1+k)(1-\nu) \gamma(k, \nu)+2 \beta(k, \nu) \Gamma\left(1+\frac{k}{2}\right)}$,

where

$\gamma(k, \nu)=\sqrt{\pi} C(k, \nu) \sin \left(\frac{\beta(k, \nu) \pi}{2}\right) \Gamma\left(\frac{1+k}{2}\right)$,

$C(k, \nu)=\frac{2^{1+k}}{\pi \Gamma(2+k)} \Gamma\left(\frac{3+k+\beta(k, \nu)}{2}\right) \times$

$\times \Gamma\left(\frac{3+k-\beta(k, \nu)}{2}\right)$,

$\beta(k, \nu)=\sqrt{(1+k)\left(1-\frac{k \nu}{1-\nu}\right)}$.

At first glance, functions (8) may seem to be complicated. However, they actually contain only one non-elementary standard gamma-function

$\Gamma(z)=\int_{0}^{\infty} t^{z-1} \mathrm{e}^{-t} \mathrm{~d} t$

and can be easily calculated by a computer.

In order to describe the tangential displacement of an indenter, we should firstly present a procedure describing its normal motion. For our further consideration to be universal, we have to select the measurement units for all quantities and to formulate the detachment criterion. This task can be fulfilled while describing the normal contact.

When the transformed profile $g(x)$ is indented into the elastic base to the depth $d$, the tensions of separate springs inside the contact can be found using the formula

$u_{z}(x)=d-g(x)=d-\frac{x^{2}}{(k+1) R}$,

${ }^{1}$ Note that the definition of the function $h_{T}(k, \nu)$ used in work [13] differs from that proposed in this work. We changed the form of this function to modify the form of Eq. (5) and write it in a form similar to Eq. (4). Of course, from the mathematical and physical viewpoints, this representation is absolutely equivalent to what was done in work [13]. 


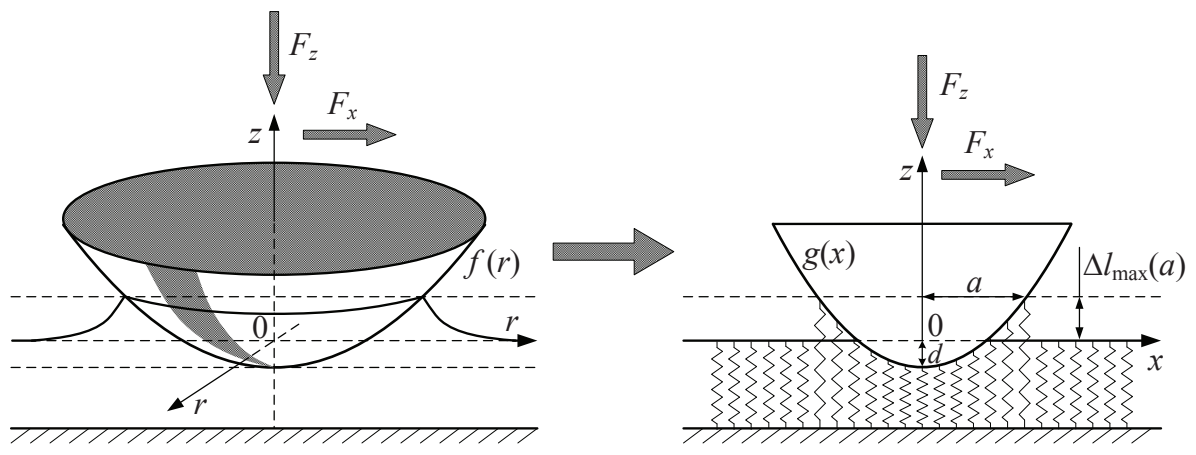

Fig. 1. MDR of transforming the original three-dimensional profile $f(r)$ into a one-dimensional profile $g(x)$ and the substitution of the elastic half-space by an elastic foundation composed of non-interacting springs. In the case of simultaneous normal and tangential forces and adhesion, the springs simultaneously shift in the normal and tangential directions. The figure illustrates only the normal motion of the indenter and the string compression in this direction

which follows from system's geometry (Fig. 1). Provided that the indentation depth $d$ is known, the size of the adhesive contact can be easily determined with the help of the virtual work principle.

The tension of the springs at the contact boundary equals $\Delta l=-u_{z}(a)$. If those two boundary springs are detached, the performed work is equal to their potential energy $k_{z}(a)(\Delta l)^{2}$. On the other hand, after the springs have been detached, there arises a free space around the contact in the form of a ring with an area of $2 \pi a \Delta x$, which is associated with an energy of $2 \pi a \Delta x \Delta \gamma$. The latter can only be determined in the original three-dimensional system. According to the virtual work principle, the system is in equilibrium, if the indicated two energies are identical [17]. From whence, we obtain

$c_{N}(a)(\Delta l)^{2} \Delta x=2 \pi a \Delta x \Delta \gamma$.

This means that the equilibrium condition for the outer springs looks like [13]

$\Delta l=\Delta l_{\max }(a)=\sqrt{\frac{2 \pi a \Delta \gamma}{c_{N}(a)}}$.

By combining expressions (10) and (12), we get [13]

$d=\frac{a^{2}}{(1+k) R}-\sqrt{\frac{2 \pi a \Delta \gamma}{c_{N}(a)}}=\frac{a^{2}}{(1+k) R}-$

$-\sqrt{\frac{2 \pi \Delta \gamma c_{0}^{k} a^{1-k}}{E^{*} h_{N}(k, \nu)}}$.

Finally, the normal force in the contact can be found by summing up the forces over all stretched and com- pressed springs [13],

$$
\begin{aligned}
& F_{z}(a)=\int^{a} c_{N}(x)[d-g(x)] \mathrm{d} x= \\
& =\frac{4 h_{N}(\bar{k}, \nu) E^{*} a^{k+3}}{c_{0}^{k}(1+k)^{2}(3+k) R}-\sqrt{\frac{8 \pi \Delta \gamma h_{N}(k, \nu) E^{*}}{a^{-(3+k)}(1+k)^{2} c_{0}^{k}}} .
\end{aligned}
$$

Now, let us consider a more general situation where the indenter simultaneously moves in the normal and tangential directions. Let us denote its tangential displacement as $u_{x}^{(0)}$. For the analytical and numerical results to be presented in the universal form, let us introduce the following dimensionless variables:

$\tilde{a}=a / a_{0}, \quad \tilde{F}_{z}=F_{z} / F_{0}, \quad \tilde{F}_{x}=F_{x} / F_{0}$,

$\tilde{d}=d / d_{0}, \quad \tilde{u}_{x}^{(0)}=u_{x}^{(0)} / d_{0}, \quad \tilde{u}_{z}=u_{z} / d_{0}$,

where $F_{0}, a_{0}$, and $d_{0}$ are the critical values of the normal force, contact radius, and absolute value of the indentation depth at the time moment, when the parabolic profile is detached from the elastic halfspace in the "fixed grips" case, respectively. In particular [13],

$$
\begin{aligned}
& F_{0}=\frac{(1-k)(5+3 k) \pi R \Delta \gamma}{2(3+k)}, \quad d_{0}=\frac{(3+k) a_{0}^{2}}{\left(1-k^{2}\right) R} \\
& a_{0}=\left(\frac{\pi\left(1-k^{2}\right)^{2} R^{2} \Delta \gamma c_{0}^{k}}{8 E^{*} h_{N}(k, \nu)}\right)^{\frac{1}{3+k}} .
\end{aligned}
$$

In the dimensionless form, expressions (13) and (14) are more compact:

$\tilde{d}=\frac{(1-k) \tilde{a}^{2}}{3+k}-\frac{4}{3+k} \sqrt{\tilde{a}^{1-k}}$,

ISSN 2071-0194. Ukr. J. Phys. 2020. Vol. 65, No. 3 

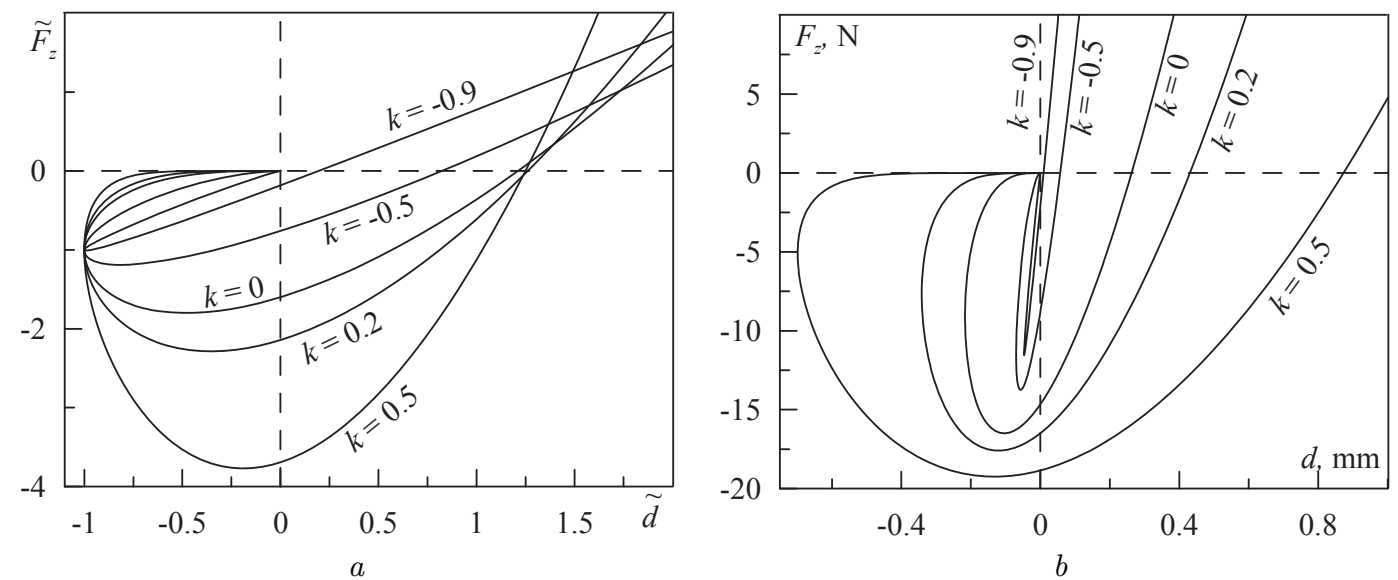

Fig. 2. Dimensionless $(a)$ and dimensional $(b)$ dependences of the elastic force $F_{z}$ on the indentation depth $d$ [see analytical formulas (17) and (18)] for the normal adhesive contact and various fixed values of the parameter $k$. The model parameters are $\Delta \gamma=0.7 \mathrm{~J} / \mathrm{m}^{2}, c_{0}=1 \mathrm{~m}, R=5 \mathrm{~m}, E=10^{6} \mathrm{~Pa}$, and $\nu=0.3$

$\tilde{F}_{z}=\frac{(1-k) \tilde{a}^{3+k}}{5+3 k}-\frac{2(3+k)}{5+3 k} \sqrt{\tilde{a}^{3+k}}$.

Of course, at $k=0$ [according to Eq. (1), if $k=0$, we have $E(z)=E_{0}=$ const], this result coincides with the classical one [18]. But in our work, we use other dimensionless variables (16), because we believe that they better suit for the further analysis.

The dependences of the dimensionless force $\tilde{F}_{z}$ on the dimensionless indentation depth $\tilde{d}$, which are determined by expressions (17) and (18), are shown in Fig. 2, $a$. The same dependences, but in the dimensional form, are depicted in Fig. 2, $b$. The selected measurement units [Eqs. (15) and (16)] allow results (13) and (14) to be written in a much simpler form [see Eqs. (17) and (18)]. The application of dimensionless equations makes the analytical analysis much easier. But sometimes, the dimensionless dependences cannot be compared at all. For example, the dimensional dependences $F(d)$ in Fig. 2, $b$ were plotted for the parameter value $c_{0}=1 \mathrm{~m}$. At the same time, this parameter enters only the measurement units but is absent from the dimensionless dependences (17) and (18). On the other hand, if we directly analyze results (13) and (14) written in terms of dimensional quantities, it is easy to see that a change of the gradientness parameter $k$ affects the adhesive contact properties differently in the cases $c_{0}>1 \mathrm{~m}$ and $c_{0}<1 \mathrm{~m}$. This means that, when analyzing the influence of this parameter, we must compare the results expressed in terms of dimensional quantities or choose the dimensionless parameters that do not contain the index $k$.

In this work, we analyze the contact stability, i.e. we determine conditions under which the contact is destroyed. In particular, loading conditions are known to be important for the adhesive contact. We consider two typical cases: the controlled force ("fixed load") and controlled displacement ("fixed grips") conditions. The controlled displacement condition means that the indenter motion corresponds to the gradual change in the macroscopic position of the indenter as a whole with the help of a very rigid connection between the indenter and the driving mechanism. The controlled force condition is realized when the indenter is moved by means of a very soft spring, when the force value does not change at every contact relaxation to the equilibrium state (the soft spring can be stretched to a rather large size; therefore, small indenter displacements do not substantially affect the spring elongation and, hence, the effective force). Earlier, we have used the MDR to simulate the influence of the adhesive interaction on collisions between elastic spherical particles under the controlled displacement condition [19].

\subsection{Simultaneous normal and tangential displacements}

Let the contact simultaneously undergo normal and tangential displacements, with the tangential displacement of the indenter being equal to $u_{x}^{(0)}$. Taking 
this quantity into account, the potential energy of two outer springs in the contact can be written in the form $k_{z}(a) u_{z}(a)^{2}+k_{x}(a) u_{x}^{(0) 2}$. By equating this energy to the adhesion work $2 \pi a \Delta x \Delta \gamma$, we arrive at the following energy balance relation:

$h_{N}(k, \nu) E^{*} u_{z}(a)^{2}+h_{T}(k, \nu) G^{*} u_{x}^{(0)^{2}}=$

$=2 \pi c_{0}^{k} a^{1-k} \Delta \gamma$

In the case $k=0$, it exactly coincides with the Johnson rule for homogeneous media $[17,20]$. From this expression, the magnitude of the equilibrium tension of outer springs can be calculated:

$\left|u_{z}(a)\right|=\sqrt{\frac{2 \pi a^{1-k} \Delta \gamma c_{0}^{k}}{h_{N}(k, \nu) E^{*}}-\frac{h_{T}(k, \nu) G^{*}}{h_{N}(k, \nu) E^{*}} u_{x}^{(0) 2}}$.

The combination of Eqs. (20) and (10) gives rise to the following relation between the indentation depth $d$ and the contact radius $a$ :

$d=\frac{a^{2}}{(1+k) R}-\sqrt{\frac{2 \pi a^{1-k} \Delta \gamma c_{0}^{k}}{h_{N}(k, \nu) E^{*}}-\frac{h_{T}(k, \nu) G^{*}}{h_{N}(k, \nu) E^{*}} u_{x}^{(0) 2}}$.

The corresponding normal and tangential forces in the contact are given by the expressions

$F_{z}=2 \int_{0}^{a} c_{N}(x)[d-g(x)] \mathrm{d} x=$

$=\frac{2 E^{*} h_{N}(k, \nu)}{c_{0}^{k}(1+k)}\left(a^{1+k} d-\frac{a^{3+k}}{(3+k) R}\right)$,

$F_{x}=2 \int_{0}^{a} c_{T}(x) u_{x}^{(0)} \mathrm{d} x=\frac{2 h_{T}(k, \nu) G^{*} u_{x}^{(0)} a^{k+1}}{c_{0}^{k}(1+k)}$,

respectively. In terms of the dimensionless variables (15), this result can be written in the more compact form,

$$
\begin{aligned}
& \tilde{d}=\frac{\tilde{a}^{2}(1-k)}{3+k}-\sqrt{\frac{16 \tilde{a}^{1-k}}{(3+k)^{2}}-\frac{h_{T}(k, \nu) G^{*}}{h_{N}(k, \nu) E^{*}} \tilde{u}_{x}^{(0) 2},} \\
& \tilde{F}_{z}=\frac{\tilde{a}^{1+k}}{2(5+3 k)}\left[\tilde{d}(3+k)^{2}-\tilde{a}^{2}\left(1-k^{2}\right)\right], \\
& \tilde{F}_{x}=\frac{h_{T}(k, \nu) G^{*}(3+k)^{2} \tilde{a}^{k+1}}{2 h_{N}(k, \nu) E^{*}(5+3 k)} \tilde{u}_{x}^{(0)} .
\end{aligned}
$$

Equations (24)-(26) describe the relation between the normal force $F_{z}$ and the indentation depth $d$. Hence, this relation depends on the tangential displacement $u_{x}^{(0)}$. Note that the substitution of expression (24) taken with the zero tangential displacement, $u_{x}^{(0)}=0$, into Eq. (25) reproduces result (18) obtained for the normal contact, with the tangential force being absent in this case, i.e. $F_{x}=0$.

Attention should be paid to that expression (19) describes a situation where the work of the adhesive forces does not depend on the indenter motion direction. This assumption may be not valid for some physical systems [21,22]. In this case, the dependence of the surface energy $\Delta \gamma$ on the angle at which the indenter moves with respect to the half-space has to be additionally taken into account, as was done in work [21] for a homogeneous medium. However, the consideration of this situation in details will significantly complicate the procedure described below. Therefore, it should be carried out in a separate study.

In what follows, we remain in the framework of the Johnson representation (19) and use equations based on it. We describe two separate cases: the controlled displacement ("fixed grips") and controlled force ("fixed load") conditions. The contact can be loaded horizontally in the framework of both of those scenarios. However, for the sake of simplicity, we only consider the case of the controlled horizontal displacement, as was done in work [17].

\subsubsection{Adhesion under the controlled force condition}

If the contact force changes gradually, there arises an instability at the time moment, when the absolute value of the negative normal force $F_{z}$ becomes maximum (see Fig. 2). This effect can be easily explained by the following example. Let us gradually increase an external force in the direction giving rise to the contact destruction. Then, for every value of this force, the established displacement $d$ corresponds to the contact force with the identical magnitude. In so doing, we move along the dependence $\tilde{F}_{z}(\tilde{d})$ in Fig. 2, a toward smaller $d$-values and larger $\left|\tilde{F}_{z}\right|^{-}$ magnitudes. When the parameter $\tilde{d}$ reaches a value at which the magnitude $\left|\tilde{F}_{z}\right|$ of the normal contact force is maximum, any growth of the external force will result in that the contact force becomes less than the external force, which will lead to a rapid destruction of the contact.

ISSN 2071-0194. Ukr. J. Phys. 2020. Vol. 65, No. 3 
A scenario where we try to tear something away is a very common case of the controlled force condition. When so doing, we have to permanently increase the applied force until it exceeds a critical value. Without making use of special instruments, we cannot control the displacements in glued contacts in the course of their destruction. We can only control the force.

In accordance with the aforesaid, the condition of contact instability in the case of controlled force looks like $\mathrm{d} \tilde{F}_{z} / \mathrm{d} \tilde{a}=0$. Differentiating Eq. (25) and making allowance for relation (24), we obtain

$$
\begin{aligned}
& \tilde{a}_{c, f l}^{2} \sqrt{\frac{16 \tilde{a}^{1-k}}{(3+k)^{2}}-\frac{h_{T}(k, \nu) G^{*}}{h_{N}(k, \nu) E^{*}} \tilde{u}_{x}^{(0) 2}}-\frac{4 \tilde{a}_{c, f l}^{1-k}}{1-k}+ \\
& +\frac{(3+k)(1+k) h_{T}(k, \nu) G^{*} \tilde{u}_{x}^{(0) 2}}{2(1-k) h_{N}(k, \nu) E^{*}}=0 .
\end{aligned}
$$

This equation describes the dependence of the critical contact radius $\tilde{a}_{c, f l}$ on the tangential displacement $\tilde{u}_{x}^{(0)}$ under the controlled force condition. The relation between the normal and tangential forces at which the contact is destroyed can be obtained by substituting the critical radius $\tilde{a}_{c, f l}$ into Eqs. (24)-(26).

\subsubsection{Adhesion under the controlled displacement condition}

If the distance between the indenter and the halfspace gradually increases, the contact between them continues to exist as long as there exists the corresponding point in the mathematical dependence $\tilde{F}_{z}(\tilde{d})$ (Fig. 2, a). To put it differently, the contact becomes destroyed when this distance becomes smaller than the plot minimum at which the condition $\mathrm{d}(\tilde{d}) / \mathrm{d} \tilde{a}=0$ is realized. This case is a little simpler than the previous one, because we have to differentiate the function $\tilde{d}(\tilde{a})$ [Eq. (24)], which explicitly depends on the contact radius $\tilde{a}$, with respect to this radius. After the differentiation, we get

$\tilde{a}_{c, f g}^{3+k}-\frac{h_{T}(k, \nu) G^{*}(3+k)^{2}\left(\tilde{u}_{x}^{(0)} \tilde{a}_{c, f g}^{1+k}\right)^{2}}{16 h_{N}(k, \nu) E^{*}}-1=0$.

This equation determines the critical radius $\tilde{a}_{c, f g}$ as a function of the tangential displacement of the indenter, $\tilde{u}_{x}^{(0)}$. The system of equations $(24)-(26)$ and (28) makes it possible to obtain expressions for the critical forces at which the contact is destroyed:

$$
\begin{aligned}
& \tilde{F}_{x}^{2}=\frac{4 h_{T}(k, \nu) G^{*}}{h_{N}(k, \nu) E^{*}}\left(\frac{3+k}{5+3 k}\right)^{2}\left(\tilde{a}_{c, f g}^{3+k}-1\right), \\
& \tilde{F}_{z}=\frac{(1-k) \tilde{a}_{c, f g}^{3+k}-2(3+k)}{5+3 k} .
\end{aligned}
$$

Their combination, after excluding the contact radius $\tilde{a}_{c, f g}$, brings about the following explicit dependence between the force components:

$\tilde{F}_{z}=\frac{h_{N}(k, \nu) E^{*}(5+3 k)(1-k)}{4 h_{T}(k, \nu) G^{*}(3+k)^{2}} \tilde{F}_{x}^{2}-1$.

Thus, in the case of controlled displacement, we derived the analytic relation, Eq. (31), between the force components at which the contact is destroyed. In the case of controlled force, Eq. (27) does not allow an analytical solution, so that a numerical analysis is required to calculate the dependence between those forces. Contact problems for gradient materials have no analytical solutions in most cases. But, sometimes, this can be done (see, e.g., work [23]), although special functions used at that have to be calculated numerically anyway.

The controlled displacement condition is realized, if the indentation depth $d$ is changed at a constant velocity. Such conditions are very often reproduced in experiments studying the adhesion, when the indenter is moved by means of an external drive characterized by a much higher stiffness than the adhesive contact itself. This configuration makes it possible to study contact phenomena in the quasistatic regime, when the drive velocity is very low and a stationary contact is realized at every time moment $[24,25]$. This is the case where the JKR theory [18] is valid. Another situation arises, if the contact characteristics are studied at various indenter velocities, when the viscoelastic properties of contacting materials substantially affect the contact phenomena $[26,27]$.

\section{Numerical Simulation and Discussion of Results}

Before proceeding to the numerical analysis of Eqs. (27) and (28) - note that, instead of numerically solving Eq. (28), we can immediately use the obtained analytical result (31) - we have to find relations for the model parameters at which the initial 


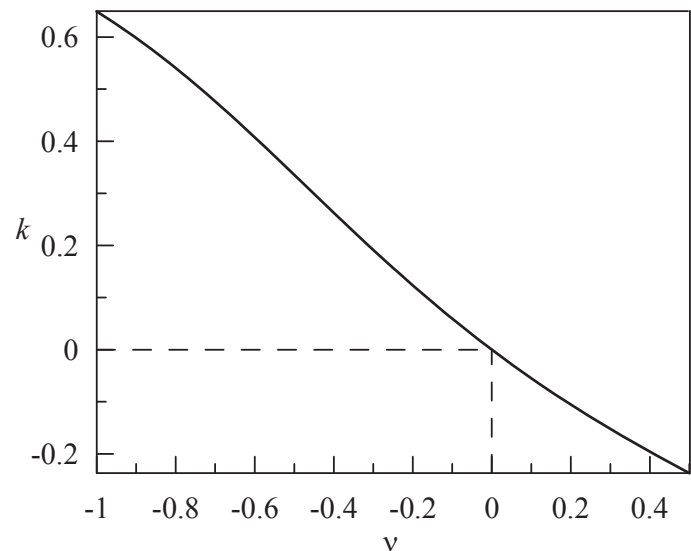

Fig. 3. Numerical solution $k(\nu)$ of Eq. (33). In the case $\nu=0$, the medium is homogeneous $(k=0)$

equation (19) has a physical meaning. In the case of homogeneous medium and at $k=0$, relation (19) reads $[17]$

$E^{*}\left[u_{z}(a)\right]^{2}+G^{*}\left[u_{x}^{(0)}\right]^{2}=2 \pi a \Delta \gamma$.

In work [17], it was shown that expression (32) is valid only in the case $\nu=0$, i.e. if $E^{*}=G^{*}$, which follows from Eq. (7). Then Eq. (32) is identical to the expression proposed in work [20]. Currently, a detachment criterion that would be correct for gradient media at $k \neq 0$ has not been formulated yet. However, Eq. (19) testifies that the tangential and normal motions give equivalent contributions to the elastic energy (as it takes place in Eq. (32) obtained in the case $\nu=0$ ), only if $h_{N}(k, \nu) E^{*}=h_{T}(k, \nu) G^{*}$. Therefore, let us focus our attention on this variant.

Taking Eq. (7) into account, the equality $h_{N}(k, \nu) E^{*}=h_{T}(k, \nu) G^{*}$ can be rewritten in the form

$$
\frac{2 \beta(k, \nu) \Gamma\left(1+\frac{k}{2}\right)}{\gamma(k, \nu)(1-\nu)}-\frac{2 \beta^{2}(k, \nu)}{1+k}+k+1=0 .
$$

The dependence $k(\nu)$ obtained by numerically solving Eq. (33) is shown in Fig. 3. It was plotted within the interval $-1<\nu \leq 0.5$ for the argument values that are characteristic of thermodynamically stable materials [28]. The figure demonstrates the relationship between the parameters $k$ and $\nu$ at which the equality $h_{N}(k, \nu) E^{*}=h_{T}(k, \nu) G^{*}$ holds true. Below, we carry out simulation calculations in which the specific values of Poisson's ratio $\nu$ are not indicated. But the corresponding value of $\nu$ can be determined from Fig. 3 for every value of $k$.

Note that, according to the obtained dependence $k(\nu)$, the problem has a solution only in the interval $-0.23688<k<0.649005$. Of course, the relation between the normal and tangential components of the forces at which the contact is destroyed must exist at any relation between $k$ and $\nu$. However, the detachment criterion has to be found for arbitrary $k$ and $\nu$ values because criterion (19) is valid only for the definite relation between those parameters (see Fig. 3). Nevertheless, we may assume that the results obtained below will describe the detachment process with a sufficient accuracy, if the values of the parameters $k$ and $\nu$ (taken in the pair) deviate only insignificantly from the curve depicted in Fig. 3.

In the framework of the MDR, we consider the normal and tangential contacts to be independent of each other. However, this assumption is correct only for certain relations between the elastic parameters. In our case - for the absolutely stiff indenter and the elastic base - the tangential and normal motions are independent, if Poisson's ratio equals $\nu=1 /(2+k)[13,29]$. But in this case, criterion (19) is not obeyed. For the further analysis, we selected such parameter values at which the detachment criterion (19) is valid, but the tangential and normal contacts were considered independently. Therefore, the results obtained below contain a certain error; however, it is insignificant [30]. For example, in the case of homogeneous medium $(k=0)$, the normal and tangential contacts are independent of each other, only if $\nu=0.5$, but, within the interval $0.3<\nu<0.5$, the error does not exceed $3 \%$ [30].

The main purpose of this work was to analyze the influence of the gradientness parameter $k$ on the ratio between the critical force components at which the contact is destroyed. Therefore, it is reasonable to plot the dependences of those forces. However, the measurement units of the force $F_{0}$ contain the parameter $k$ [see Eq. (16)]. Therefore, when comparing the dependences $\tilde{F}_{z}\left(\tilde{F}_{x}\right)$, one cannot speak about the influence of the gradientness parameter on system's behavior. The measurement units (16) are very convenient, because they made it possible to derive universal formulas in which the forces are normalized by the critical values. But, following this way, it is difficult to trace the role of the gradientness parameter

ISSN 2071-0194. Ukr. J. Phys. 2020. Vol. 65, No. 3 

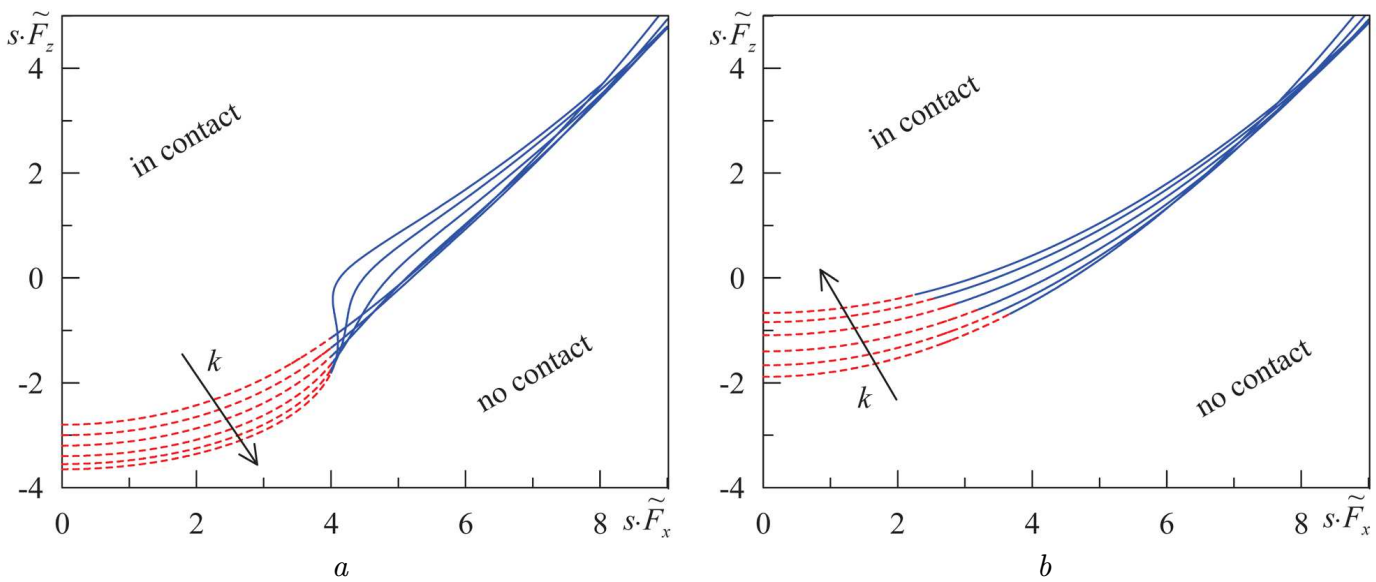

Fig. 4. Dependences of the normalized critical normal force component $s \tilde{F}_{z}$ on the normalized tangential force component $s \tilde{F}_{x}$, which satisfy the condition $h_{N}(k, \nu) E^{*}=h_{T}(k, \nu) G^{*}$, for various $k=-0.2,0.0,0.2,0.4,0.55$, and 0.649005 (the growth of this parameter is indicated by arrows). Panel $a$ corresponds to the conditions of controlled force in the vertical direction and controlled displacement in the horizontal one, and panel $b$ to the condition of controlled displacement in both directions. Dashed sections correspond to the indenter detachment, when the indenter is located above the half-space and the indentation depth $d$ is negative; solid sections correspond to the indenter detachment at positive values of the indentation $d$ into the half-space

$k$. Therefore below, we will not analyze the dependence $\tilde{F}_{z}\left(\tilde{F}_{x}\right)$. Instead, we will consider the dependence $s \tilde{F}_{z}\left(s \tilde{F}_{x}\right)$ with the parameter

$s=\frac{(1-k)(5+3 k)}{(3+k)}$.

According to expressions (15) and (16), the coefficient $k$ does not enter the formula for the connection between forces $s \tilde{F}_{z}\left(s \tilde{F}_{x}\right)$. This fact means that, by analyzing such dependences for various values of the parameter $k$, one can trace the influence of the latter on the ratio between the critical values of force components.

Figure 4 illustrates the dependences $s \tilde{F}_{z}\left(s \tilde{F}_{x}\right)$ for the critical force components which were calculated under the controlled force (panel $a$ ) and controlled displacement (panel $b$ ) conditions. The dependences were obtained for various values of the parameter $k$ describing the gradientness degree of the material. Let us consider the dashed sections of the curves. They correspond to the case where the contact is destroyed, if there is a gap between the indenter and the half-space, i.e. the indentation depth $d$ has a negative value. For these dashed sections of the curves corresponding to the controlled force condition (Fig. 4, a), the adhesive strength of the contact increases with the parameter $k$. It is so because the larger tangential and/or normal forces have to be applied in order to destroy the contact at larger $k$ values. However, the controlled displacement condition (Fig. 4, b) brings about a different situation. Namely, the strength of the adhesive contact decreases, as the parameter $k$ increases. The critical values of the forces at which the contact becomes destroyed in the absence of a tangential displacement $\left(\tilde{F}_{x}=0\right)$ can be written in the form [13]

$s \tilde{F}_{z}^{c, f l}=-(k+3), \quad s \tilde{F}_{z}^{c, f g}=-s$

for both loading types. Here, $s$ is determined by expression (34), and the superscripts $f l$ and $f g$ denote the controlled force (fixed load) and controlled displacement (fixed grips) conditions, respectively.

Formulas (35) correspond to the starting point (absence of a tangential displacement, $\tilde{F}_{x}=0$ ) of the dependences shown in Fig. 4. The values of critical forces given by Eqs. (35) coincide only at $k=-1$. In all other cases, the absolute value of $s \tilde{F}_{z}^{c, f l}$ at $\tilde{F}_{x}=0$ always exceeds $s \tilde{F}_{z}^{c, f g}$ for the relevant values of the parameter $k(k>-1)$. Moreover, we consider an even narrower interval of $k$-values: $-0.23688<k<$ $<0.649005$ (see the figure caption to Fig. 3).

It is of interest that the solid sections of the curves exhibited in Fig. 4 change their relative positions with respect to each other at large values of the tangential force. As a result, if the indentation depth and the tangential displacement are large, it is impossi- 


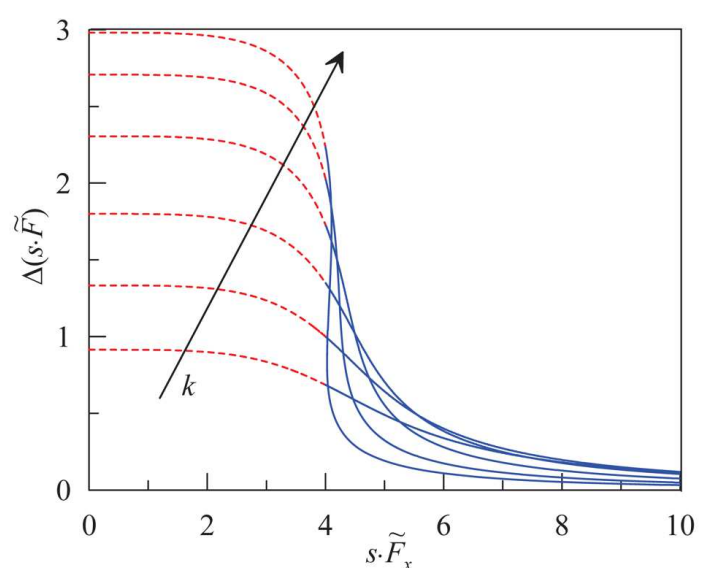

Fig. 5. Dependences of the difference between the normal and tangential force components shown in Fig. 4 on the normalized tangential force $s \tilde{F}_{x}$. All curves correspond to the dependences shown in Fig. 4. The growth of the parameter $k$ is indicated by an arrow

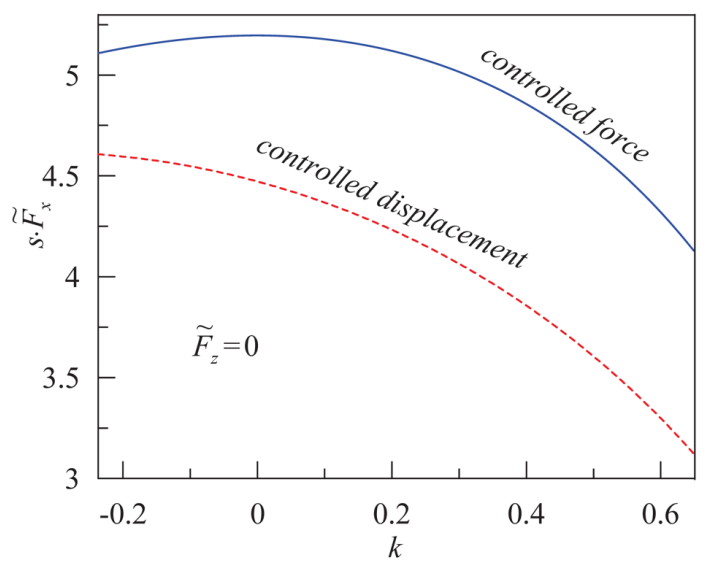

Fig. 6. Dependences of the critical value of the tangential force $s \tilde{F}_{x}$ on the parameter $k$ at the zero normal force component, $\tilde{F}_{z}=0$, under the controlled force (solid curve, the parameters of Fig. 4, $a$ ) and controlled displacement (dashed curve, the parameters of Fig. 4,b) conditions

ble to say surely which material (which $k$-value) will favor the maximum strength of the adhesive contact, so that additional calculations are required in this case. However, as a rule, more interesting for the application problems is the variant shown by dashed curves in Fig. 4. In this case, the influence of the parameter $k$ can be clearly determined from the plotted dependences.

In Fig. 4, $a$, a non-monotonic dependence between the force components is observed at large $k$ - values. Let us consider this peculiarity in more details. Any curve in either of two panels in the figure divides the space of force values into two regions. For a fixed $k$-value, the areas to the left and right from any curve correspond to the existence and absence of the contact, respectively. The curve itself corresponds to the condition, when the contact becomes unstable. In the case of monotonic $\tilde{F}_{z}\left(\tilde{F}_{x}\right)$ dependence (Fig. 4, b), the situation is quite clear: if we start to move in the area of stable contact and reach, in that or another way, the area of instability, the contact is destroyed.

However, if the dependence between the force components is non-monotonic, a different scenario is possible. Namely, let us move from the stability area. In a certain interval of the tangential force $\tilde{F}_{x}$ values, we enter firstly the region of unstable contact, and the latter becomes destroyed. Afterward, we enter back into the stability region. But in this scenario, the contact is not reproduced, because we consider a situation where the adhesive bonds do not recover after having been broken [17]. However, if the adhesive contact can be restored after the destruction of some of its part and the relaxation of elastic stresses that arose at this site, another regime also becomes possible. More specifically, it is a regime with a fixed tangential force, when the contact firstly becomes completely destroyed at the detachment stage and, afterward, recovers again. This is a rather interesting result. However, in order to describe this situation, it is necessary to consider the restoration of adhesive bonds, as was done in work [31]. On the other hand, the described non-monotonic dependence opens an opportunity to perform such manipulations with the contact at which the latter, provided the monotonic dependence $\tilde{F}_{z}\left(\tilde{F}_{x}\right)$, would be completely destroyed. But, in the non-monotonic case, it would be possible to vary the tangential force, thus avoiding this non-monotonic feature and, as a result, preserving the contact.

Figure 5 demonstrates the dependences $\Delta(s \tilde{F})$ for the difference $\Delta$ between the normal forces under the controlled displacement and controlled force conditions, which were shown in Fig. 4. Each difference was determined at a fixed value of the tangential force $s \tilde{F}_{x}$. From Fig. 5 , it follows that the indicated difference decreases with the growth of the tangential force. A similar behavior was also observed in our previous work [17]. Figure 5 testifies that the differ- 
ence between the forces calculated for different loading conditions also diminishes with a decrease of the parameter $k$. Note that we consider the values of the parameter $k$ that fall within the interval shown in Fig. 3. An analogous calculation of the $\Delta \tilde{F}$ value at $k=-1$ gives the result $\Delta \tilde{F}=0$ because, as was already indicated above, the dependences $\tilde{F}_{z}\left(\tilde{F}_{x}\right)$ corresponding to different loading conditions completely coincide at this $k$-value.

In this work, we study the influence of a tangential displacement on the adhesive contact strength. Here, there appears an interesting scenario, when the indenter is detached under the action of a tangential displacement only, i.e. when the normal force $\tilde{F}_{z}$ equals zero. The dependences of the critical tangential force on the parameter $k$ at the zero normal force under different loading conditions are shown in Fig. 6. One can see that, under the controlled displacement condition, the strength of a tangential contact decreases with increasing the gradientness index $k$. But if the force is controlled, when the indenter moves, the adhesive strength of the contact increases together with the growth of the negative values of this parameter. Then, when $k$ becomes positive, the adhesive strength begins to decrease as the parameter $k$ grows further. The same result follows from Fig. 4.

\section{Conclusions}

In this paper, the strength of adhesive contacts in the presence of a tangential displacement is studied for gradient materials in which the elasticity modulus varies with the depth according to the power law. It is found that a change in the gradientness index of the material substantially affects the shear resistance of the contact. Accordingly, materials that will provide the strongest contact are indicated. Two types of loading are considered: the controlled force and the controlled displacement. An interesting result is obtained, which consists in that the dependence between the critical forces has a hysteretic character in the controlled force case, which allows several scenarios for the contact destruction to take place depending on the contact prehistory. Note that we consider the situation where the adhesive bonds are not restored after the destruction, and the destroyed part of the adhesive contact does not contribute to the tangential force component. This assumption is true, if the surface energy significantly decreases at the sites, where the adhesion bonds have been broken. Such a scenario can be observed, e.g., in glued contacts or in the contacts whose adhesive strength increases considerably in time.

The work was supported by the Deutsche Forschungsgemeinschaft (project DFG PO 81055-1) and the Ministry of Education and Science of Ukraine in the framework of the grant from the President of Ukraine supporting the research of young scientists "Transition mode between adhesion and sliding in tangential dynamic contact for normal and gradient materials" 0119 U103175 (contest F82 of the State Fund for Fundamental Research of Ukraine).

1. Functionally Graded Materials: Design, Processing and Applications. Edited by Y. Miyamoto, W.A. Kaysser, B.H. Rabin, A. Kawasaki, R.G. Ford (Springer, 1999) [ISBN: 978-1-4615-5301-4].

2. A. Gupta, T. Mohammad. Recent development in modeling and analysis of functionally graded materials and structures. Prog. Aerosp. Sci. 79, 1 (2015).

3. I. Argatov, A. Iantchenko. Rayleigh surface waves in functionally graded materials - long-wave limit. Q. J. Mech. Appl. Math. 72, 197 (2019).

4. F. Jin, X. Guo, W. Zhang. A unified treatment of axisymmetric adhesive contact on a powerlaw graded elastic halfspace. J. Appl. Mech. 80, 061024 (2013).

5. J. Aboudi, M.-J. Pindera, S.M. Arnold. Higher-order theory for functionally graded materials. Compos. Part B Eng. 30, 777 (1999).

6. M. Hill, R. Carpenter, G. Paulino, Z. Munir, J. Gibeling. Fracture testing of a layered functionally graded material. In Fracture Resistance Testing of Monolithic and Composite Brittle Materials, edited by J. Salem, G. Quinn, M. Jenkins (ASTM International, 2002), p. 169.

7. C.-E. Rousseau, V.B. Chalivendra, H.V. Tippur, A. Shukla. Experimental fracture mechanics of functionally graded materials: An overview of optical investigations. Exp. Mech. 7, 845 (2010).

8. V.L. Popov, R. Pohrt, Q. Li. Strength of adhesive contacts: Influence of contact geometry and material gradients. Friction 5, 308 (2017).

9. E. Martinez-Paneda, R. Gallego. Numerical analysis of quasi-static fracture in functionally graded materials. Int. J. Mech. Mater. Des. 11, 405 (2015).

10. Q. Li, V.L. Popov. Boundary element method for normal non-adhesive and adhesive contacts of power-law graded elastic materials. Comp. Mech. 61, 319 (2017).

11. Q. Li, R. Pohrt, I.A. Lyashenko, V.L. Popov. Boundary element method for nonadhesive and adhesive contacts of a coated elastic half-space. Proc. Inst. Mech. Eng. J. 234, 73 (2019).

12. V.L. Popov, M. Hess. Method of dimensionality reduction in contact mechanics and friction: a user's handbook. I. Axially-symmetric contacts. FU Mech. Eng. 12, 1 (2014). 
13. M. Hess, V.L. Popov. Method of dimensionality reduction in contact mechanics and friction: a user's handbook. II. Power-law graded materials. FU Mech. Eng. 14, 251 (2016).

14. M. Hess. A simple method for solving adhesive and non-adhesive axisymmetric contact problems of elastically graded materials. Int. J. Eng. Sci. 104, 20 (2014).

15. E. Willert, A.I. Dmitriev, S.G. Psakhie, V.L. Popov. Effect of elastic grading on fretting wear. Sci. Rep. 9, 7791 (2019).

16. I. Argatov. From Winkler's foundation to Popov's foundation. FU Mech. Eng. 17, 181 (2019).

17. V.L. Popov, I.A. Lyashenko, A.E. Filippov. Influence of tangential displacement on the adhesion strength of a contact between a parabolic profile and an elastic half-space. Roy. Soc. Open Sci. 4, 161010 (2017).

18. K.L. Johnson, K. Kendall, A.D. Roberts. Surface energy and the contact of elastic solids. Proc. R. Soc. Lond. A 324, 301 (1971).

19. I.A. Lyashenko, E. Willert, V.L. Popov. Adhesive impact of an elastic sphere with an elastic half space: Numerical analysis based on the method of dimensionality reduction. Mech. Mat. 92, 155 (2016).

20. K.L. Johnson. Adhesion and friction between a smooth elastic spherical asperity and a plane surface. Proc. $R$. Soc. Lond. A 453, 163 (1997).

21. I.A. Lyashenko. Tangential displacement influence on the critical normal force of adhesive contact breakage in biological systems. FU Mech. Eng. 14, 313 (2016).

22. J.W. Hutchinson, Z. Suo. Mixed mode cracking in layered materials. Adv. Appl. Mech. 29, 63 (1991)

23. E. Willert. Dugdale-Maugis adhesive normal contact of axisymmetric power-law graded elastic bodies. FU Mech. Eng. 16, 9 (2018).

24. W. Deng, H. Kesari. Depth-dependent hysteresis in adhesive elastic contacts at large surface roughness. Sci. Rep. 9, 1639 (2019).

25. B.N.J. Persson. Adhesion between an elastic body and a randomly rough hard surface. Eur. Phys. J. E 8, 385 (2002).
26. Z. Liu, H. Lu, Y. Zheng, D. Tao, Y. Meng, Y. Tian. Transient adhesion in a non-fully detached contact. Sci. Rep. 8, 6147 (2018).

27. M. Scaraggi, D. Comingio. Rough contact mechanics for viscoelastic graded materials: The role of small-scale wavelengths on rubber friction. Int. J. Solids Struct. 125, 276 (2017).

28. L.D. Landau, E.M. Lifshitz. Theory of Elasticity (Pergamon Press, 1970) [ISBN: 9780080064659].

29. D.L. Holl. Stress transmission in earths. Highway Res. Board Proc. 20, 709 (1940).

30. F.M. Borodich, B.A. Galanov, Y.I. Prostov, M.M. SuarezAlvarez. Influence of complete sticking on the indentation of a rigid cone into an elastic half space in the presence of molecular adhesion. J. Appl. Math. Mech. 76, 590 (2012).

31. V.L. Popov, A.V. Dimaki. Friction in an adhesive tangential contact in the Coulomb-Dugdale approximation. J. Adhes. 93, 1131 (2017).

Received 04.09.19.

Translated from Ukrainian by O.I. Voitenko

Я.О. Ляшенко, З.М. Ляшенко

ВПЛИВ ТАНГЕНЦІАЛЬНОГО ЗСУВУ НА СИЛУ АДГЕЗІї МІЖ ГРАДІЄНТНИМИ МАТЕРІАЛАМИ

$\mathrm{P}$ е $з$ ю м е

Досліджується вплив тангенціального зміщення на міцність адгезійного контакту для градієнтних матеріалів із різним ступенем градієнтності. Розглядаються умови контрольованої сили і контрольованого зміщення. Для всього діапазону параметра градієнтності, для якого справедливий критерій відриву, розраховане співвідношення між критичними нормальною і тангенціальною компонентами сили, за яких відбувається руйнування контакту. Знайдено оптимальні параметри, при яких міцність адгезійного контакту набуває максимальних значень. Окремо розглянуто випадок, у якому контакт руйнується лише за рахунок тангенціального зміщення, коли нормальна сила набуває нульового значення. 